Paidéia, 2003, 13(25); 73-84

\title{
COMPETÊNCIA INTERPESSOAL, PROBLEMAS ESCOLARES E A TRANSIÇÃO DA MENINICE À ADOLESCÊNCIA ${ }^{1}$
}

\author{
Marli Aparecida Silva Campos \\ Universidade Estadual Júlio de Mesquita \\ Edna Maria Marturano ${ }^{2}$ \\ FMRP - Universidade de Säo Paulo
}

\begin{abstract}
RESUMO: Este artigo focaliza adolescentes com experiência prévia de insucesso escolar. Seu objetivo foi verificar se crianças com queixas escolares, avaliadas por suas mães como competentes no domínio interpessoal quando admitidas a uma clínica de psicologia, mostram na adolescência melhor desempenho e ajustamento, quando comparadas a outros da mesma população clínica. Participaram 48 adolescentes, com idade entre 10 e 15 anos. Com base em indicadores de competência interpessoal extraídos dos prontuários da clínica foram formados três grupos: Competente; Vulnerável; de Referência. Em entrevista de seguimento, feita de um a dois anos após a alta, os participantes foram avaliados mediante Teste de Desempenho Escolar, Escala Infantil Piers-Harris de Auto-Conceito, Roteiro de Auto-Eficácia, entrevista de seguimento e CBCL. Comparações entre grupos indicaram que os adolescentes anteriormente avaliados como competentes apresentam melhores resultados de desempenho, auto-percepções e comportamento. Recursos interpessoais parecem associados a trajetórias mais favoráveis.
\end{abstract}

Palavras-chave: competência interpessoal; desempenho escolar; auto-percepção; adolescência inicial

\section{INTERPERSONAL COMPETENCE, SCHOOL PROBLEMS AND TRANSITION FROM CHILDHOOD TO ADOLESCENCE}

\begin{abstract}
This paper focuses on adolescents who had a previous experience of school failure. Its main purpose was to verify whether children with academic complains but judged by their mother as interpersonally competent would show better achievement and adjustment later, when compared with others of the same clinical population. Forty eight adolescents aged 10 and 15 years participated. On the basis of interpersonal skills indices obtained from the clinic archive records, three groups were formed: Competent; Vulnerable; Reference. At a follow-up one to two years after clinical discharge, children were assessed with the Academic Achievement Test, the Piers-Harris Children's Self-Concept Scale, the Self-Efficacy Schedule, a follow-up interview and the CBCL. In group comparisons, the adolescents that were classified as interpersonally competent in childhood had better academic, self-perception and behavior results. These results suggest that, in face of school adversities, children with better interpersonal resources have more favorable developmental trajectories.
\end{abstract}

Keywords: interpersonal competence; academic achievement; self-perception; early adolescence

Naș sociedades ocidentais, adolescência é um termo genérico para designar o conjunto de transformações biológicas e psicossociais que ocorrem durante a segunda década da vida. Essa longa transi-

\footnotetext{
${ }^{1}$ Artigo recebido para publicação em 27/01/03; aceito em 20/05/03

2 Endereço para correspondência: Edna Maria Marturano, Departamento de Neurologia, Psiquiatria e Psicologia Médica, Faculdade de Medicina de Ribeirão Preto, USP, Av. Nove de Julho, 980, Ribeirão Preto, SP, 14025-000, E-mail: emmartur @ fmrp.usp.br
}

ção entre a meninice e a idade adulta freqüentemente é vista como um período inerentemente difícil, durante o qual o desenvolvimento saudável está mais relacionado ao fato de evitar problemas que ao crescimento de competências. A crença de que o desenvolvimento do adolescente normal é tumultuoso constitui um dos vários mitos a respeito dessa fase (Offer \& Schonert-Reichl, 1992).

Essa noção tem apoio limitado em evidências 


\section{Marli Aparecida.Silva Campos}

de pesquisas. Com efeito, há particularidades na adolescência que aumentam a probabilidade de comportamentos de risco (Mrazek \& Haggerty, 1994), Achados epidemiológicos sugerem uma elevação na taxa de distúrbio entre a meninice $e$ a adolescência, situando em aproximadamente $20 \%$ a parcela de adolescentes que têm problemas de saúde mental e necessitam de ajuda (Offer \& Schonert-Reichl, 1992). Entretanto, é preciso lembrar que a maior parte dos jovens - cerca de $80 \%$ deles - passa por este período do desenvolvimento sem maiores problemas.

Segundo Steinberg e Morris (2001), a ênfase dada pelos pesquisadores ao comportamento problema resultou em achados que contribuem hoje para relativizar a antiga concepção da adolescência como um período tormentoso. Problemas persistentes muitas vezes precedem a adolescência. Desse modo, eles não podem ser debitados a esse período do desenvolvimento. Um exemplo disso são os problemas de conduta iniciados na pré- escola. (Fergusson, Lynskey \& Horwood, 1996).

A argumentação de Steinberg e Morris relembra que os padrões adaptativos na adolescência não podem ser compreendidos sem considerar o desenvolvimento prévio do indivíduo, já que problemas que eclodem na adolescência freqüentemente têm sua origem em fases anteriores. Como um período de transição no desenvolvimento, é essencial ver a adolescência através de uma perspectiva de curso da vida, atentando para os caminhos do desenvolvimento que emergiram anteriormente, e que podem resultar em sucesso ou insucesso no enfrentamento dos desafios da fase (Cichetti \& Rogosch, 2002).

Neste texto, aspectos da adaptação do adolescente são examinados, no período inicial da fase, compreendido entre 11 e 15 anos. O ponto de vista adotado é o de que o grau de competência do adolescente para lidar com os desafios inerentes à fase atual resulta, em parte, do grau de sucesso na resolução dos desafios de etapas anteriores.

Desenvolvimento como processo organizador da experiência individual e a adaptação durante a adolescência

A perspectiva teórica aqui adotada concebe o desenvolvimento como um processo contínuo de re- organização e integração ativa, pelo indivíduo, das diversas competências adquiridas ao longo da vida. O desenvolvimento é caracterizado por uma integração hierárquica de sistemas de comportamento, através da qual as estruturas mais antigas são incorporadas a estruturas mais recentes, sob formas cada vez mais complexas. O indivíduo participa ativamente deste processo, trazendo para as novas experiências as atitudes, expectativas e sentimentos derivados de uma história de interações que, por seu lado, influenciam a maneira como as pistas e os estímulos ambientais são interpretados e organizados (Egeland, Carlson \& Sroufe, 1993).

Nesse enfoque, a competência é vista como a habilidade de usar com sucesso recursos internos e externos para resolver questões que são proeminentes no estágio de desenvolvimento em que o indivíduo se encontra. A competência para resolver questões em um período de desenvolvimento não prediz necessariamente a competência posterior; em vez disso, pensa-se que a competência em um período torna o indivíduo, em sentido amplo, adaptado ao ambiente e preparado para desenvolver competência no período seguinte. (Sroufe \& Rutter, 1984).

O desenvolvimento compreende um conjunto de tarefas relevantes para cada estágio no ciclo da vida, definidas de acordo com os contextos culturais em que o indivíduo se desenvolve. Entretanto, uma vez cumpridas, as tarefas não perdem importância. Embora sua saliência possa declinar, diminuir em relação a questões que vão emergindo, as tarefas permanecem importantes para a adaptação ao longo do tempo. A resolução bem sucedida de uma questão relevante em um estágio do desenvolvimento aumenta a probabilidade de ajustamento bem sucedido em etapas posteriores (Sroufe \& Rutter, 1984).

Uma tarefa central da adolescência é desenvolver um senso de si como um indivíduo autônomo. O impulso para tal autonomia deriva dos processos internos e biológicos que fazem a transição para um papel mais adulto (puberdade e maturidade cognitiva crescente), e das mudanças nos papéis e expectativas sociais que acompanham essas transformações fisiológicas e cognitivas (Eccles, 1999).

Os recursos internos de que o adolescente dispõe para resolver as tarefas de desenvolvimento importantes dessa fase vão depender significativamen- 
te da qualidade da resolução das tarefas relevantes da meninice, que incluem ajustamento e desempenho escolar, competência nas relações com os companheiros e conduta governada por regras, no sentido de aceitação das normas da sociedade para comportamento moral e conduta pró-social (Masten \& Coatsworth, 1998). Para o adolescente inicial que foi capaz de alcançar resoluções competentes frente às tarefas dos estágios anteriores, a organização interna dos domínios de desenvolvimento serão mais flexíveis e integradas, e haverá maior capacidade adaptativa disponível para o esforço de resolver os desafios do período. Em contraste, crianças que não conseguem progresso no aprendizado escolar ou que se mantêm impulsivas, agressivas ou socialmente incompetentes estão em alto risco para distúrbios psicossociais na adolescência (Mrazek \& Haggerty, 1994). Entre esses extremos, os indivíduos vão variar quanto à adaptabilidade de sua organização interna, que compreende as competências evolutivas construídas no curso do desenvolvimento anterior.

\section{Dificuldades escolares e o percurso da menini- ce à adolescência}

Este artigo trata de trajetórias de desenvolvimento da meninice à adolescência, em crianças com vivência de insucesso escolar precoce. No pressuposto de que o grau de competência do adolescente para lidar com os desafios inerentes à fase atual resulta, em parte, do grau de sucesso na resolução dos desafios de etapas anteriores, o desempenho escolar pobre na meninice pode contribuir tanto para a vulnerabilidade como para a competência do adolescente.

Por um lado, a criança que não progride na escola pode desenvolver sentimentos de baixa autoestima e inferioridade (Erikson, 1971), com repercussões desfavoráveis no desenvolvimento. Investigando as relações entre auto-conceito, senso de autoeficácia e desempenho escolar em crianças com dificuldade de aprendizagem, Jacob (2001) verificou que essas crianças têm auto-percepções desfavoráveis quando comparadas a crianças com desempenho escolar satisfatório. É amplamente reconhecida a relação de co-ocorrência entre baixo rendimento escolar e problemas de comportamento (Hinshaw, 1992).
Segundo Roeser e Eccles (2000), uma das vias pelas quais essa associação se estabelece é aquela das autopercepções. As avaliações que as crianças fazem de sua dificuldade escolar podem contribuir indiretamente para a instalação de problemas sócio-emocionais, através do impacto de tais avaliações sobre as auto-percepções da criança, que estão em desenvolvimento na fase escolar. Entre as auto-percepções relevantes para esse processo estão aquelas relativas a competência acadêmica, valor acadêmico e crenças sobre o apoio dos outros em situações de aprendizagem. Crenças sobre competência, valores e percepção de suporte efetivo estão associadas a sentimentos de estima, interesse e pertença. Inversamente, sentimentos de incompetência, menos valia e pouco suporte estão associados a sentimentos de vergonha e dúvida, desinteresse e isolamento (Roeser \& Eccles, 2000).

Por outro lado, o insucesso escolar na meninice parece não afetar negativamente o desenvolvimento quando a criança está enfrentando com sucesso as demais tarefas da fase, que estão estreitamente ligadas à competência interpessoal. Assim, crianças que são de fácil contato com o outro, respeitam as regras e normas socialmente instituídas evoluem favoravelmente, mesmo em presença de dificuldades escolares persistentes, como sugere um estudo recente de Santos (1999). Essa autora seguiu a trajetória de vida de adolescentes que haviam apresentado dificuldade no aprendizado escolar durante a meninice. Através de roteiros de entrevistas, obteve indicadores de recursos e vulnerabilidade pessoais e ambientais, nos dois momentos da vida dos participantes. Seus resultados indicaram que dificuldades interpessoais na meninice foram preditoras de dificuldades interpessoais e problemas de saúde mental na adolescência, ao passo que a competência interpessoal mostrou associação positiva com um bom ajustamento global posterior em crianças com queixas escolares. Os resultados do estudo de Santos sugerem que dificuldades interpessoais precoces da criança são precursoras de dificuldades interpessoais e acadêmicas na adolescência, enquanto recursos no domínio interpessoal na meninice são precursores de competência, tanto interpessoal como acadêmica, nos anos posteriores. É provável que nesses diferentes percursos entrem em ação processos transacionais 


\section{Marli Aparecida Silva Campos}

que, com efeitos cumulativos, estabelecem para cada indivíduo estilos de interação coerentes nos diversos contextos de desenvolvimento, com continuidade no tempo. A criança envolvida em interações mutuamente satisfatórias tende a fortalecer seu senso de competência através dás pistas positivas que recebe das pessoas significativas do seu entorno; a criança com dificuldades interpessoais tem probabilidade crescente de envolver-se em interações disfuncionais, que a tornam mais vulnerável frente aos desafios emergentes da adolescência (Olson, Bates, Sandy \& Lanthier, 2000). Segundo Papalia e Olds (2000) pesquisas recentes sobre o desenvolvimento da auto-estima na meninice têm demonstrado que a percepção de apoio social pode ser um componente mais importante que a percepção do sucesso acadêmico para a formação de uma imagem positiva de si.

\section{Objetivo}

A investigação relatada neste artigo retoma o tema a partir do estudo de Santos (1999), ampliando seu foco para inciuir a avaliação de auto-percepções, um aspecto não abordado por aquela autora. $\mathrm{A}$ competência interpessoal em crianças que enfrentaram o problema do baixo desempenho escolar em um período sensível de suas vidas é associada a indicadores de desempenho e ajustamento na adolescência inicial. Foram estudadas crianças que na meninice foram avaliadas por seus cuidadores como incompetentes na tarefa de desenvolvimento relacionada ao domínio escolar, e percebidas seja como competentes ou incompetentes nas tarefas relacionadas ao domínio interpessoal. Embora não tenham sido testados mecanismos específicos envolvendo as relações entre desempenho escolar e competência interpessoal, o estudo foi conduzido sob o pressuposto de que recursos interpessoais poderiam atenuar manifestações comumente associadas ao fracasso escolar, como problemas de comportamento, baixo auto-conceito e baixo senso de auto-eficácia.

Seu objetivo específico foi verificar se crianças com queixas escolares, avaliadas por suas mães como competentes no domínio interpessoal por ocasião da admissão a uma clínica de psicologia, mostram posteriormente, na adolescência inicial, melhor desempenho escolar, auto-percepções mais favorá- veis e menos problemas de comportamento, quando comparadas a outros adolescentes que também têm história prévia de insucesso escolar mas foram avaliados, na meninice, como crianças menos competentes ou até mesmo vulneráveis no domínio interpessoal. Procurou-se alcançar o objetivo através de um estudo de seguimento.

\section{Método}

\section{Local}

O estudo foi realizado em uma clínica-escola de Psicologia vinculada à rede SUS de atendimento, em uma cidade de aproximadamente 500 mil habitantes no interior do Estado de São Paulo.

\section{Participantes}

Participaram quarenta e oito adolescentes de ambos os sexos e suas respectivas mães. Todos tinhiam sido referidos à clínica em razão do desempenho escolar pobre; passado por atendimento psicopedagógico e recebido alta clínica. Por ocasião do seguimento, suas idades variavam entre 10 e 15 anos.

\section{Instrumentos}

Os instrumentos utilizados foram o Teste de Desempenho Escolar (Stein, 1994), a Escala Infantil Piers-Harris de Auto Conceito (Jacob, 2001), o Roteiro de Auto-eficácia (Medeiros, 2000), um roteiro de entrevista de seguimento (Santos, 1999) e o CBCL - Inventário de Comportamentos da Infância e Adolescência (Achenbach, 1991; Bordin, Mari \& Caeiro, 1995).

\section{Coleta de dados}

Para composição da amostra, foram localizados, no arquivo da clínica, os prontuários de crianças que ingressaram entre 1996 e 1998. Para inclusão na amostra, a criança deveria ter permanecido até o final do atendimento, recebendo alta clínica, e o momento da alta deveria ter ocorrido entre 1998 e 1999. À medida que eram localizados os prontuários nessa 
condição, a primeira autora entrava em contato com as famílias, agendando entrevistas de seguimento. Estas foram feitas entre 2000 e 2001, ou seja, dois a quatro anos após a admissão na clínica e pelo menos um ano após a alta.

Nessas entrevistas, mãe e criança entravam na sala de atendimentos e os objetivos da pesquisa eram explicados para ambos. Após a leitura do termo de consentimento para que pudessem participar da pesquisa, o mesmo era assinado pela mãe, caso concordassem em participar. Em seguida, procedia-se a entrevista com a criança para aplicação das técnicas. A entrevista com a mãe acontecia com a aplicação do Roteiro de Entrevista de Seguimento e do CBCL.

\section{Composição dos grupos}

Mediante a leitura dos relatos das entrevistas de admissão, feitas com as mães por ocasião do ingresso na clínica, as informações sobre características de cada participante nos relacionamentos com adultos e crianças foram classificadas de acordo com os seguintes indicadores: (a) recursos: fácil relacionamento com adultos, fácil relacionamento com crianças, respeito a re- gras e normas; (b) dificuldades: problemas nos relacionamentos, agressividade, desrespeito a regras e normas. Com base na combinação de critérios relativos a recursos e a dificuldades, foram formados três grupos: G1, crianças competentes $(n=17)$, que apresentavam, segundo o relato da mãe, os três indicadores de recursos e nenhum indicador de dificuldade no domínio interpessoal; $\mathrm{G} 2$, crianças vulneráveis $(n=15)$, com os três indicadores de dificuldade e nenhum indicador de recurso no domínio interpessoal; $G 3$, grupo de referência, $(n=16)$, constituído de crianças que apresentavam tanto recursos como dificuldades, porém em menor número que os participantes dos demais grupos. $\mathrm{O}$ procedimento de composição dos grupos foi conduzido pela segunda autora, que não participou da coleta de dados.

A Tabela 1 apresenta a caracterização dos participantes quanto a sexo, idade e escolaridade por ocasião do seguimento. Através de ANOVAs e teste $X$, não foram encontradas diferenças estatisticamente significativas entre os grupos nessas variáveis, nem em relação ao nível cognitivo registrado no prontuário por ocasião da admissão na clínica.

Tabela 1: Caracterização dos participantes quanto a sexo, idade e escolaridade por ocasião do seguimento

\begin{tabular}{llllll}
\hline & Variável & G1 & G2 & G3 & Total \\
\hline Sexo & Masculino & 13 & 08 & 09 & 30 \\
& Feminino & 04 & 07 & 07 & 18 \\
Idade no seguimento & & & & \\
$10-12$ anos & 9 & 9 & 9 & 27 \\
$13-15$ anos & 8 & 6 & 7 & 21 \\
Escolaridade no seguimento & & & & \\
$1^{\mathrm{a}}-4^{\mathrm{a}}$ série & 03 & 06 & 03 & 12 \\
$5^{\mathrm{a}}$ série & 02 & 02 & 02 & 06 \\
$6^{\mathrm{a}}$ série & 09 & 06 & 08 & 23 \\
$7^{\mathrm{a}}$ série & 02 & 01 & 02 & 05 \\
$8^{\mathrm{a}}$ série & 01 & - & 01 & 02 \\
\hline
\end{tabular}

\section{Análise dos dados}

Os protocolos foram cotados de acordo com as proposições de cada técnica. Características dos três grupos no seguimento foram comparadas através de ANOVAs ou testes não paramétricos, a fim de verificar diferenças nos indicadores de desempenho escolar, auto-percepções e problemas de comportamento.
Com base nos relatos encontrados nos prontuários e nas entrevistas de seguimento, foi feito um resumo, para cada criança, das condições de ajustamento relatadas pela mãe por ocasião da admissão no ambulatório, na alta e na entrevista de seguimento. Nesse resumo foram considerados em separado os contextos: familiar, do grupo de companheiros e 


\section{Marli Aparecida Silva Campos}

escolar. Os indicadores de ajustamento procurados nos prontuários foram aqueles relativos às relações interpessoais e ao desempenho escolar. Uma condição limitante desta análise foi a heterogeneidade dos relatos. Em três prontuários (dois de G2 e um de G3) não havia relato das condições da criança na alta ${ }^{1}$.

\section{Resultados}

Comparações preliminares entre sexos indicaram diferenças significativas no escore total da Escala Piers-Harris de Auto-conceito $(t=3,01, p<0,01)$, assim como nos escores de auto-conceito relativos às categorias Comportamento $(t=3,28, p<0,01)$, Ansiedade $(t=2,90, p<0,01)$, e Aparência $(t=4,48, p$ $<0,0001)$. Em todas essas comparações as meninas mostraram auto-conceito menos favorável que os meninos. Nas demais variáveis não foram encontradas diferenças entre sexos. Mediante esses resultados, a variável sexo foi incluída como um segundo fator nas ANOVAs realizadas sobre as medidas de auto-conceito.

\section{Auto-conceito}

Nà Tabela 2 são apresentados os dados relativos à avaliação geral do auto-conceito dos grupos, bem como das seis categorias de auto-conceito. A ANOVA indicou efeitos principais de sexo e grupo nas categorias Escore Total, Comportamento e Aparência. Nenhum efeito de interação sexo $X$ grupo foi detectado.

Tabela 2: Auto-conceito: média, desvio padrão, resultados da ANOVA e comparação post-hoc pelo teste de Bonferroni.

\begin{tabular}{|c|c|c|c|c|c|c|c|c|}
\hline \multirow[t]{2}{*}{ Categoria } & \multicolumn{3}{|c|}{ Média } & \multicolumn{3}{|c|}{ Desvio padrão } & \multirow[t]{2}{*}{$\mathbf{F}$} & \multirow[t]{2}{*}{ Comparação } \\
\hline & G1 & G2 & G3 & G1 & G2 & G3 & & \\
\hline Escore total & 66,00 & 50,06 & 54,00 & 6,86 & 13,47 & 14,86 & $7,63^{* * *}$ & $\begin{array}{l}\mathrm{G} 1>\mathrm{G} 2 \\
\mathrm{G} 1>\mathrm{G} 3\end{array}$ \\
\hline Status Intelectual & 13,65 & 10,07 & 11,81 & 1,20 & 3,08 & 3,49 & $4,33^{*}$ & $\mathrm{G} 1>\mathrm{G} 2$ \\
\hline Comportamento & 13,41 & 9,93 & 11,12 & 2,57 & 4,11 & 3,48 & $6,08 * *$ & $\mathrm{G} 1>\mathrm{G} 2$ \\
\hline Ansiedade & 10,29 & 7,13 & 6,81 & 2,54 & 2,77 & 3,08 & $2,80 \dagger$ & - \\
\hline Aparência & 10,59 & 8,33 & 9,19 & 1,87 & 3,15 & 3,06 & $7,78^{* * *}$ & $\begin{array}{l}\mathrm{G} 1>\mathrm{G} 2 \\
\mathrm{G} 1>\mathrm{G} 3\end{array}$ \\
\hline Popularidade & 8,82 & 7,07 & 7,50 & 1,91 & 2,91 & 3,16 & 1,88 & - \\
\hline Felicidade & 9,23 & 7,53 & 7,56 & 0,83 & 1,92 & 1,89 & $6,02 * *$ & $\begin{array}{l}\mathrm{G} 1>\mathrm{G} 2 \\
\mathrm{G} 1>\mathrm{G} 3\end{array}$ \\
\hline
\end{tabular}

${ }^{*} \mathrm{p}<0,05 ;{ }^{* *} \mathrm{p}<0,01 ;{ }^{* * *} \mathrm{p}=0,001 ; \dagger \mathrm{p}<0,10$

Em relação ao auto-conceito, os participantes de G1, grupo competente, obtiveram escores mais elevados, com diferença estatisticamente significativa,

\footnotetext{
' Aspectos Éticos:O projeto foi aprovado pelo Comitê de Ética em Pesquisa da instituição onde foi conduzido. Todas as mães participantes assinaram o Termo de Consentimento Livre e Esclarecido. Tratando-se de uma amostra de ex-clientes de um serviço de psicologia, alguns procedimentos éticos específicos foram adotados. Uma vez concluída a coleta de dados com a família, era feita uma avaliação clínica do ajustamento atual da criança, com base nos dados fornecidos e nas observações e impressōes colhidas durante o contato direto. Quando a autora julgava haver necessidade de acompanhamento clínico, ela se reunia novamente com a mãe e a criança, procedendo uma devolução dos resultados e encaminhamento para serviços na comunidade. Foram encaminhadas onze crianças - nove do $\mathrm{G} 2$ e duas do $\mathrm{G3}$
}

compatíveis com um auto conceito global mais positivo, comparativamente aos resultados dos demais grupos. $\mathrm{Na}$ análise das categorias de auto-conceito, os participantes de G1 apresentaram maiores médias, em relação aos participantes de G2, nas categorias Comportamento, Status Intelectual e Acadêmico, Aparência, Felicidade. Nas categorias Aparência e Felicidade, G1 apresentou também média significativamente mais alta que $\mathrm{G} 3$.

\section{Auto-eficácia}

$\mathrm{Na}$ comparação entre os grupos, os participantes de G1 tiveram escore total significativamente 
mais elevado, compatível com uma avaliação de autoeficácia mais positiva quando comparados aos participantes de G2 e G3, que apresentaram valores inferiores, sugestivos de uma auto avaliação menos favorável $(F=10,65, p<0,0001)$. Nas comparações por itens, através do Teste Exato de Fischer, o grupo competente mostrou, em relação ao grupo de referência, escores significativamente mais favoráveis de autoeficácia nos itens: Eu tenho me saído bem (e seu reverso, Eu tenho me saído mal), Eu consigo copiar com facilidade, Eu acabo as atividades no mesmo tempo que os colegas. O grupo vulnerável não apresentou nenhuma diferença em relação ao grupo de comparação na análise por item. Por outro lado, quando comparado ao grupo competente, teve auto-avaliações mais desfavoráveis em nove dos 20 itens do
Roteiro de Avaliação de Auto-eficácia, incluindo todos os itens que discriminaram G1 e G3 e mais os seguintes: Eu consigo escrever as palavras que são ditadas, Eu demoro mais que os outros para acabar as atividades, Eu esqueço rápido o que aprendi, Eu quero continuar estudando por muitos anos, Eu quero parar de estudar logo.

\section{Desempenho escolar}

As informações referentes aos resultados do Teste de Desempenho Escolar encontram-se na Tabela 3. Os resultados são expressos em termos de clássificação do desempenho, obtida de acordo com as normas do teste, comparando-se o escore bruto de cada participante com o esperado para sua série.

Tabela 3: Classificação das crianças dos três grupos no TDE, em relação à série cursada.

\begin{tabular}{llccc}
\hline \multirow{2}{*}{ Variável } & Classificação do & \multicolumn{3}{c}{ Grupos } \\
\cline { 3 - 5 } & desempenho & G1 & G2 & G3 \\
\hline Escrita & Superior & 5 & - & - \\
& Médio & 02 & 01 & 03 \\
& Inferior & 10 & 14 & 13 \\
\hline Aritmética & Superior & - & - & - \\
& Médio & 06 & - & 01 \\
& Inferior & 11 & 15 & 15 \\
\hline Leitura & Superior & 14 & 07 & 12 \\
& Médio & 02 & 03 & 02 \\
& Inferior & 01 & 05 & 02 \\
\hline Inferior & Superior & - & - & - \\
& Médio & 08 & 01 & 02 \\
& & 09 & 14 & 14 \\
\hline
\end{tabular}

Os dados da Tabela 3 sugerem que nos três grupos há crianças com desempenho aquém do esperado para a série que cursam, principalmente em relação à aritmética e menos em relação à leitura. Essa tendência é mais acentuada no Grupo 2 e menos pronunciada no Grupo 1.

Para a comparação estatística entre os grupos, a classificação no TDE foi pontuada da seguinte forma: superior $=3$, médio $=2$ e inferior $=1$. Os grupos foram comparados através do teste não paramétrico $U$ de Mann-Whitney. Os resultados indicaram que no subteste Escrita não houve diferenças entre os grupos. No subteste Aritmética, as cri- anças de G1 mostraram desempenho superior às de $G 2(p=0.024)$. Neste subteste, a diferença entre $G 1$ e $\mathrm{G} 3$ tendeu à significância $(\mathrm{p}=0,09)$. No subteste Leitura, o desempenho das crianças de G1 foi superior ao das crianças de $\mathrm{G} 2(\mathrm{p}=0,028)$. Finalmente, na classificação relativa ao desempenho total no TDE, foram observadas diferenças significativas entre $\mathrm{Gl}$ e G2 ( $p=0,013)$ e entre G1 e G3 $(p=0,034)$, sempre favoráveis a $\mathrm{Gl}$.

\section{Comportamento}

A ocorrência de problemas de comportamento foi avaliada a partir de informações fornecidas 


\section{Marli Aparecida Silva Campos}

pelas mães, através do $\mathrm{CBCL}$. A análise focalizou o escore total e os escores nas oito síndromes que compõem o perfil de problemas de comportamento fornecido pelo inventário. Seguindo uma recomendação do autor (Achenbach, 1991) relativa ao uso dos resultados das síndromes do $\mathrm{CBCL}$ em pesquisa, a análise foi feita sobre os escores brutos, e não sobre os escores normalizados (escores $\mathrm{T}$ ). Os resultados encontram-se na Tabela 4.

Tabela 4: Inventario de Comportamentos da Infância e da Adolescência: média, desvio padrão, resultados da ANOVA e comparação post-hoc pelo teste de Bonferroni.

\begin{tabular}{|c|c|c|c|c|c|c|c|c|}
\hline \multirow{2}{*}{ Síndrome } & \multicolumn{3}{|c|}{ Média } & \multicolumn{3}{|c|}{ Desvio padrão } & \multirow[t]{2}{*}{$\mathbf{F}$} & \multirow[t]{2}{*}{ Comparação } \\
\hline & G1 & G2 & G3 & G1 & G2 & $\mathbf{G 3}$ & & \\
\hline Retraimento & 6,65 & $\overline{8.0}$ & 6.31 & 2.98 & 4.21 & 3.74 & 0.92 & - \\
\hline Queixas Somáticas & 3,23 & 4,0 & 0,81 & 4.28 & 4,10 & 1.56 & $3,47^{*}$ & $\mathrm{G} 2>\mathrm{G} 3$ \\
\hline Ansiedade /Depressão & 7,29 & 10,93 & 9,0 & 3,77 & 5,62 & 4,32 & $2,50 \dagger$ & $\mathrm{G} 2>\mathrm{G} 1$ \\
\hline Problemas Sociais & 4,29 & 7,80 & 4,56 & 2,31 & 3,99 & 2,37 & $6,78 * *$ & $\begin{array}{l}\mathrm{G} 2>\mathrm{G} 1 \\
\mathrm{G} 2>\mathrm{G} 3\end{array}$ \\
\hline Problemas de Pensamento & 1,88 & 3,07 & 1,50 & 1,76 & 3,63 & 1,09 & 1,83 & 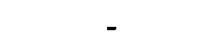 \\
\hline Problemas de Atenção & 6,94 & 11,27 & 8,18 & 4,60 & 3,49 & 3,81 & $4,81 *$ & $\mathrm{G} 2>\mathrm{G} 1$ \\
\hline Comportamento delinqüente & 2,88 & 4,47 & 4,50 & 2,54 & 3,83 & 2,98 & 1,43 & n. s. \\
\hline Comportamento agressivo & 11,82 & 19,93 & 14,31 & 7,00 & 8,46 & 7,19 & $4,76^{*}$ & $\mathrm{G} 2>\mathrm{G} 1$ \\
\hline Total de problemas & 52,65 & 79,13 & 57,31 & 19,35 & 29,65 & 20,51 & $5,69 * *$ & $\begin{array}{l}\mathrm{G} 2>\mathrm{G} 1 \\
\mathrm{G} 2>\mathrm{G} 3\end{array}$ \\
\hline
\end{tabular}

$* 0,05 ;{ }^{* *} \mathrm{p}<0,01 ; \uparrow \mathrm{p}<0,10$

De acordo com os resultados apresentados na tabela, não foram encontradas diferenças relativas a problemas de comportamento entre o grupo competente e o grupo de referência. Nas comparações entre grupos, é o grupo vulnerável que se destaca, apresentando mais problemas emocionais e de comportamento em relação aos demais. Comparado ao grupo de referência, mostra média significativamente mais alta nos escores de queixas somáticas e problemas sociais. Em relação ao grupo competente, diferenças foram encontradas nos eixos ansiedade / depressão, problemas sociais, problema de atenção e comportamento agressivo.

\section{Evolução clínica}

O Quadro 1 apresenta o resumo da evolução dos participantes a partir de sua situação inicial no momento da admissão ao atendimento na clínica. O número de participantes de cada grupo sobre os quais essa análise foi possível está indicado entre parênteses.

Analisando-se a amostra como um todo, verifica-se que, segundo o relato das mães, todas as cri- anças apresentavam melhora, por ocasião da alta, nas dificuldades escolares. Por outro lado, dentre as crianças que apresentavam dificuldades nas relações interpessoais, melhoras nesse aspecto foram relatadas em cerca de metade dos casos. Comparando-se a evolução entre a alta e o seguimento, nota-se que na maior parte dos casos de melhora esta se mantém. Entretanto, em nenhum caso se observa melhora entre a alta e o seguimento.

Cotejando-se a evolução dos casos nos três grupos, pode-se dizer que o Grupo 2 é o que menos melhoras apresenta na alta e também o que menos mantém as melhoras no seguimento. Comparandose os resultados relativos à manutenção dos ganhos obtidos no desempenho escolar, observa-se uma gradação entre os três grupos, com aproveitamento total no grupo competente e a menor taxa de manutenção no grupo vulnerável. Com relação às dificuldades interpessoais, verifica-se que no grupo de referência estas são menos freqüentes e menos homogêneas que no grupo vulnerável, porém, quando presentes, tendem a se manter ou reaparecer em proporção semelhante. 
Quadro 1: Evolução dos participantes entre o momento da admissão, a alta clínica e a entrevista de seguimento

\begin{tabular}{|c|c|c|c|c|}
\hline \multirow[b]{2}{*}{ Grupo } & \multirow[b]{2}{*}{ Contexto } & \multicolumn{3}{|c|}{ Situação relatada pela mãe } \\
\hline & & Admissão & Alta & Seguimento \\
\hline \multirow[t]{3}{*}{$\begin{array}{l}\text { Competente } \\
(\mathrm{n}=17)\end{array}$} & Famńlia & Todos estão bem & $\begin{array}{l}\text { Todos continuam } \\
\text { bem }\end{array}$ & $\begin{array}{l}\text { Todos continuam } \\
\text { bem }\end{array}$ \\
\hline & Pares & Todos estão bem & $\begin{array}{l}\text { Todos continuam } \\
\text { bem }\end{array}$ & $\begin{array}{l}\text { Todos continuam } \\
\text { bem }\end{array}$ \\
\hline & Escola & Todos têm dificuldade & Todos melhoram & $\begin{array}{l}\text { Todos mantêm a } \\
\text { melhora }\end{array}$ \\
\hline \multirow[t]{3}{*}{$\begin{array}{l}\text { Vulnerável } \\
(\mathbf{n}=13)\end{array}$} & Família & $\begin{array}{l}\text { Todos vivenciam } \\
\text { conflitos }\end{array}$ & Seis melhoram & $\begin{array}{l}\text { Dois mantêm a } \\
\text { melhora }\end{array}$ \\
\hline & Pares & $\begin{array}{l}\text { Todos se envolvem em } \\
\text { brigas }\end{array}$ & Cinco melhoram & $\begin{array}{l}\text { Três mantêm a } \\
\text { melhora }\end{array}$ \\
\hline & Escola & Todos têm dificuldade & Todos melhoram & $\begin{array}{l}\text { Cinco mantêm a } \\
\text { melhora }\end{array}$ \\
\hline \multirow[t]{3}{*}{$\begin{array}{l}\text { Referência } \\
(n=15)\end{array}$} & Família & $\begin{array}{l}\text { Seis apresentam alguma } \\
\text { dificuldade }\end{array}$ & $\begin{array}{l}\text { Todos os que tinham } \\
\text { dificuldade } \\
\text { melhoram }\end{array}$ & $\begin{array}{l}\text { Dois mantêm a } \\
\text { melhora }\end{array}$ \\
\hline & Pares & $\begin{array}{l}\text { Dois apresentam alguma } \\
\text { dificuldade }\end{array}$ & $\begin{array}{l}\text { As dificuldades } \\
\text { iniciais continuam }\end{array}$ & $\begin{array}{l}\text { As dificuldades } \\
\text { iniciais continuam }\end{array}$ \\
\hline & Escola & Todos têm dificuldade & Todos melhoram & $\begin{array}{l}\text { Dez mantêm a } \\
\text { melhora }\end{array}$ \\
\hline
\end{tabular}

\section{Síntese dos resultados}

Verifica-se, em síntese, melhor adaptação psicossocial atual no grupo de crianças competentes, tanto nos aspectos acadêmicos quanto nos comportamentais e nas auto-percepções.

Os resultados relativos ao auto-conceito e ao senso de auto-eficácia são sugestivos de que as crianças mais competentes no domínio interpessoal têm uma percepção de si e de seus atributos mais positiva, identificando em si melhores condições comportamentais e intelectuais, julgando-se mais capazes para enfrentar a realidade acadêmica. Essas diferenças nas autopercepções de competência acadêmica encontram correlato na avaliação objetiva do desempenho escolar, pelo menos quanto ao escore total, no qual as crianças mais competentes tiveram melhor resultado.

Com relação ao comportamento, o grupo que mais se diferencia é o das crianças que já apresentavam anteriormente dificuldades de relacionamento e agressividade. Este grupo mostrou índices mais elevados de problemas de comportamento no $\mathrm{CBCL}$.

Tendo como padrão de comparação o grupo de referência, as crianças competentes no domínio interpessoal mostraram melhores resultados posteri- ormente, na adolescência inicial, em indicadores globais de desempenho escolar, auto-conceito, senso de auto-eficácia e problemas de comportamento. Suas auto-concepções relativas à felicidade e aparência são mais elevadas. Contudo, nas síndromes do CBCL, o grupo competente não difere do grupo de referência.

O grupo de crianças vulneráveis não diferiu significativamente do grupo de referência nos escores totais dos instrumentos, mas se mostrou mais prejudicado que este em relação ao grupo competente, em todos os indicadores globais.

Entre o grupo competente e o grupo vulnerável foram encontradas diversas diferenças, além daquelas nos escores totais, já mencionadas. O grupo competente apresenta auto-conceito mais elevado com relação ao status intelectual e acadêmico, comportamento, aparência e felicidade; melhor classificação do desempenho em aritmética e leitura; menos problemas sociais, de atenção e agressividade no CBCL; e uma menor tendência a ansiedade / depressão.

\section{Discussão}

$\mathrm{O}$ presente trabalho investigou, em crianças referi- 


\section{Marli Aparecida Silva Campos}

das para atendimento psicológico em razão do baixo desempenho escolar, associações entre indicadores de competência ou vulnerabilidade interpessoal na meninice e indicadores de ajustamento nos anos iniciais da adolescência. Foi feito um estudo de seguimento em que os recursos ou dificuldades das crianças atendidas em uma clínica de psicologia foram associados, após um a dois anos do atendimento, com indicadores de desempenho escolar, problemas de comportamento, auto-conceito e senso de auto-eficácia. Através da análise dos relatos das mães foram formado três grupos, sendo um constituído de crianças com indicadores de competência interpessoal, um segundo com crianças apresentando problemas generalizados nos relacionamentos e um terceiro, formado por crianças com indicadores tanto de competência como de dificuldades interpessoais.

Um dado importante é que as crianças foram consideradas como tendo recursos ou dificuldades interpessoais através da percepção das mães. Os resultados das comparações entre grupos sugerem correspondência entre a percepção da mãe por ocasião do ingresso na clínica e a forma como a criança se percebe posteriormente, no seguimento. Em outras palavras, crianças percebidas por suas mães como socialmente competentes tendem a se perceber como mais ajustadas socialmente, quando avaliadas em um momento posterior. Pode-se supor que as percepções maternas orientam seu próprio comportamento em relação à criança e são de algum modo comunicadas à criança nos contatos cotidianos, contribuindo assim para a formação do autoconceito. Conforme enfatizado na introdução, a percepção de apoio das pessoas significativas pode ser um componente mais importante que a percepção do sucesso acadêmico para a formação de uma imagem positiva de si durante a meninice (Papalia \& Olds, 2000).

As diferenças encontradas entre os grupos, indicando melhor funcionamento no grupo previamente identificado como socialmente competente, vão ao encontro dos resultados da investigação precedente de Santos (1999), que relacionou competência interpessoal na meninice com melhor ajustamento global e escolar na adolescência. Os resultados da presente investigação, ao demonstrarem associação entre competência interpessoal e melhor adaptação psicossocial posterior em três domínios - ajustamento comportamental, desempenho acadêmico e auto- percepções - conferem consistência e especificidade aos achados anteriores de Santos (1999).

Embora os resultados de ambas as pesquisas sejam apenas sugestivos e não conclusivos, devido a limites metodológicos, eles apoiam a interpretação de mecanismos promotores do desenvolvimento, relacionados à competência interpessoal, propiciando trajetórias mais positivas na transição entre a meninice e a adolescência.

À parte os resultados relevantes para os objetivos do estudo, outros achados merecem reflexão quanto a seu possível significado, particularmente no que diz respeito à efetividade do tratamento psicológico realizado. No que se refere ao desempenho escolar, verifica-se que no TDE os adolescentes do grupo competente e do grupo de referência se situam, em maioria, no nível médio ou superior de leitura, porém se classificam no patamar inferior de escrita $\mathrm{e}$ aritmética. Esses resultados aparentemente contradizem as informações sobre evolução, em que as mães de ambos os grupos relatam a manutenção das melhoras escolares na maioria dos casos.

A contradição é de fato aparente, jâ que o suporte psicopedagógico clínico tem um alvo limitado que é a remoção de barreiras à aprendizagem. Como o esquema recomendado para programas de recuperação de leitura é de sessões diárias individuais (Clay, 1991), não seria de se esperar uma recuperação ao nível da série no esquema clínico semanal. Os adolescentes dos grupos 1 e 3 que alcançaram níveis de leitura compatíveis com sua série provavelmente o fizeram por efeito do suporte recebido nos sistemas familiar e escolar, uma vez superadas, com o tratamento clínico, as barreiras que os impediam de progredir no aprendizado acadêmico.

Algumas considerações devem ser feitas em relação às limitações metodológicas do presente estudo. Em primeiro lugar, o delineamento da pesquisa não permitiu uma análise precisa dos processos em jogo. Assim, interpretações a respeito de processos envolvendo as variáveis que foram alvo do estudo devem ser vistas como extrapolações dos dados, sugeridas pelos resultados em consonância com a literatura. Em outras palavras, o que os dados mostraram foi um conjunto de associações sugestivas, e não indicativas, de determinados processos e mecanismos.

Em segundo lugar, o indicador de sociabilida- 
de adotado foi derivado de informações contidas em prontuários de um serviço de saúde, com todas as limitações inerentes aos registros dessa natureza, feitos para finalidades outras que não de pesquisa.

Em terceiro lugar, o estudo se ateve a aspectos do indivíduo, não incluindo em seu modelo de análise variáveis do contexto. Para uma visão abrangente do curso do desenvolvimento seria necessária uma abordagem compreensiva, em que tanto os processos de risco e proteção presentes, quanto a história de suas transações e como eles influenciaram o indivíduo no curso do desenvolvimento anterior fossem examinados (Cichetti \& Rogosch, 2002).

Apesar dessas limitaçōes, o procedimento de composição dos grupos se mostrou adequado aos propósitos do estudo. Foi sensível para discriminar grupos de crianças que mostram cada qual um perfil e percursos de vida coerentes com as previsões feitas. Desse modo, parece que, mesmo com as restrições apontadas, a definição de critérios de competência e vulnerabilidade interpessoal foi compatível com as proposições do estudo e se mostrou produtiva.

Nesse sentido, os resultados foram coerentes com o ponto de vista teórico que guiou a investigação. Tanto no caso dos adolescentes competentes, como no caso daqueles que se encontram fragilizados, os dados sugerem continuidade entre precursores de enfrentamento na meninice e características do funcionamento atual. Os resultados são compatíveis com a visão corrente das relações interpessoais como contextos cruciais tanto para o desenvolvimento sadio, como para a formação de distúrbios e a prevenção destes. Em uma ampla gama de pontos de vista, as relações interpessoais têm um papel central na etiologia, manutenção, prevenção e remediação de distúrbios sócio-emocionais (Sroufe, Duggal, Weinfield \& Carlson, 2000).

Nesse contexto, o presente estudo terá trazido sua contribuição ao indicar como competentes nas relações interpessoais crianças que de fato mostraram, quando avaliadas por outros meios e em momento posterior, trajetórias de desenvolvimento claramente mais favoráveis que seus pares não identificados como competentes, confirmando as previsões derivadas da teoria, de que crianças sociáveis estariam mais protegidas frente à experiência fragilizante do insucesso escolar.

Através de que mecanismos se mantêm os pro- cessos protetores? Estudos sobre suporte social sugerem que pessoas competentes no domínio interpessoal têm maior probabilidade de manter e mobilizar redes de relacionamentos que lhes dêem suporte nos momentos difíceis (Burhmester, Furman, Wittenberg \& Reis, 1988). Provavelmente, a capacidade desses indivíduos para estabelecer e manter relações interpessoais mutuamente satisfatórias lhes garante um efetivo suporte dos pais, professores, outros adultos e colegas frente às adversidades da vida e contribui para um positivo senso de eficácia no enfrentamento dessas adversidades. Em um contexto interpessoal com tais características, a experiência pontual de insucesso escolar, mesmo em uma fase do desenvolvimento em que o indivíduo está particularmente vulnerável a essa experiência, tem alta probabilidade de ser superada; uma vez superada, em lugar de fragilizar, pode contribuir para of fortalecimento do senso de competência frente a desafios futuros. Foi demonstrado neste estudo que os adolescentes com maiores recursos no domínio interpessoal na meninice apresentam hoje mais recursos também no domínio acadêmico. Essa ampliação de vantagem em relação às crianças do grupo de referência parece estar de acordo com a concepção apresentada na introdução deste artigo, de que a competência em um período torna o indivíduo, em sentido amplo, adaptado ao ambiente e preparado para desenvolver competência no período seguinte (Sroufe \& Rutter, 1984).

\section{Referências Bibliográficas}

Achenbach, T.M. (1991). Manual for the Child Behavior Check-list / 4-18 and 1991 profile. Burlilngton, VT. University of Vermont Department of Psychiatry.

Bordin, I.A.S., Mari, J.J. \& Caieiro, M.F. (1995). Validação da versão brasileira do Child Behavior Checklist (CBCL) Inventário de comportamentos da infância e adolescência: dados preliminares. Revista da ABP - APPAL, 17, 55-66.

Burhmester, D., Furman, W., Wittenberg, M.T. \& Reis, H.T. (1988). Five domains of interpersonal competence in peer relationships. Journal of Personality and Social Psychology, 55, 9911008. 


\section{Marli Aparecida Silva Campos}

Cichetti, D. \& Rogosch, F.A. (2002). A Developmental Psychopathology Perspective on Adolescence. Journal of Consulting and Clinical Psychology, 70, 6-20.

Clay, M.M. (1991). Reading recovery: a guidebook for teachers in training. Auckland: Heinemann.

Eccles, J.S. (1999). The development of children ages 6 to 14. The Future of Children, 9, 30-44.

Egeland, B., Carlson. E., \& Sroufe, L.A. (1993). Resilience as process. Development and Psychopathology, 5, 517-528.

Erikson, E. (1971). Infância e sociedade. (G. Amado, Trad.) Rio de janeiro: Zahar

Fergusson, D.M., Lynskey, M.T. \& Horwood, L.J. (1996). Factors associated with continuity and changes in disruptive behavior patterns between childhood and adolescence. Journal of Abnormal Child Psychology, 24, 533-553.

Hinshaw, S.P. (1992), Externalizing behavior problems and academic underachievement in childhood and adolescence: causal relationships and underlying mechanisms. Psychological Bulletin, $111,127-155$.

Jacob, A.V. (2001). O desempenho escolar e sua relações com autoconceito e auto-eficácia. Tese de Doutorado, Universidade de São Paulo, Ribeirão Preto.

Masten, A.S. \& Coatsworth, J.D. (1998). The developmental of competence in favorable and unfavorable environments - Lessons from research on successful children. American Psychologist, $53,205-220$.

Medeiros, P.C. (2000). Auto-eficácia e aspectos comportamentais de crianças com dificuldade de aprendizagem. Dissertação de Mestrado, Universidade de São Paulo, Ribeirão Preto.

Mrazek, P.J. \& Haggerty, R.J. (1994). Reducing risks for mental disordiers: frontiers for preventive intervention research. Committee on Prevention of Mental Disorders, Division of Biobehavioral Sciences and Mental Disorders, Institute of Medicine. Washington: National
Academy Press.

Offer, D. \& K. A. Schonert-Reichl (1992). Debunking the myths of adolescence: findings from recent research. Journal of the American Academy of Child and Adolescent Psychiatry, 31, 1003-1014.

Olson, S.L., Bates, J.E., Sandy, J.M. \& Lanthier, R. (2000). Early developmental precursors of externalizing behavior in middle childhood and adolescence. Journal of Abnormal Child Psychology, 28, 119-133.

Papalia, D.E. \& Olds, S. W. (2000). Desenvolvimento Humano. Porto Alegre: Artes Médicas.

Roeser, R.W. \& Eccles, J.S. (2000). Schooling and mental health. Em A.J. Sameroff, M. Lewis \& S.M. Miller (Orgs.), Handbook of developmental psychopathology (pp. 135-156). New York: Kluwer Academic / Plenum.

Santos, L.C. (1999). Crianças com dificuldade de aprendizagem :estudo de seguimento. Dissertação de Mestrado, Universidade de São Paulo, Ribeirão Preto.

Sroufe, L.A. \& Rutter, M.R. (1984). The domain of developmental psychopathology. Child Development; 55, 17-29.

Sroufe, L.A., Duggal, S., Weinfield, N. \& Carlson, E. (2000). Relationships, development, and psychopathology. Em A.J. Sameroff, M. Lewis \& S.M. Miller (Orgs.), Handbook of developmental psychopathology (pp. 75-91). $2^{\mathrm{a}}$ ed., New York: Kluwer Academic / Plenum.

Stein, L.M. (1994). TDE-Teste de desempenho escolar: manual para aplicação e interpretação. São Paulo: Casa do psicólogo.

Steinberg, L. \& Morris, A.S. (2001). Adolescent Development. Annual Review of Psychology, 52, 83-110.

Apoio financeiro CAPES e FAPESP - trabalho derivado da dissertação de Mestrado de Marly Aparecida Silva Campos, no PPG em Psicologia da FFCLRP, USP; dados colhidos no Ambulatório de Psicologia Infantil do HC FM RP USP. 